\title{
Application of fine structure interpretation and reservoir prediction technology in Beier Sag
}

\author{
Bin Zhang ${ }^{1, \mathrm{a}}$ \\ ${ }^{1}$ Exploration and Development Research Institute, Daqing Oilfield Company Limited, Daqing, Heilongjiang, China
}

\begin{abstract}
After years of exploration in Beier Sag, the exploration is becoming more and more difficult.Beier Sag is characterized by many faults and complex structures.For a long time, the exploration and development effect of many fault blocks is not good due to unclear structure and poor reservoir prediction effect.Therefore, through the close combination of Geology and earthquake, we need to use the fine structure interpretation technology to interpret the seismic data carefully, and on this basis, we need to carry out the fine characterization of favorable reservoirs through the appropriate reservoir prediction technology.The fusion frequency inversion technology is based on improving the resolution through different inversion methods, combining with dominant frequency fusion, applying simulated annealing inversion and geostatistics (SMI) inversion to calculate separately, then fusing the inversion results of dominant frequency, and interpreting the fusion results, and finally accurately predicting the reservoir distribution in the fan body,favorable exploration targets should be implemented.
\end{abstract}

\section{Introduction}

Beier Sag experienced multi-stage tectonic movement, and the fault system is very complex, mainly characterized by small fault block area, dense fault distribution and cross cutting;it is difficult to determine the location of breakpoint and fault combination;the quality of seismic data is poor, all kinds of interferences are serious, and the target layer has weak in-phase axis reflection and poor continuity; the occurrence of the strata changes violently and it is difficult to close the strata.The strata of Nantun Formation are characterized by multi source, short source, near source, obvious phase change, overlapping of multiple fans and complex spatial relationship.The reflection characteristics of different types of fan bodies in Beier Sag are also different from each other. At the same time, due to the strong shielding effect of reflection formed by regional unconformity, the characterization of multi-stage fan bodies has become a difficult problem to be solved.

\section{Fine structural interpretation}

\subsection{Horizon calibration technology}

Using the drilling and logging data in the area, select the wells with long logging section, good data quality, complete target layers and can control the whole area to make synthetic records. Combined with the lithologic characteristics, regional seismic wave group characteristics and well geological stratification information, comprehensively calibrate the corresponding reflection layer on the seismic section. According to the polarity and dominant frequency of the wavelet extracted from the sidetrack and the seismic data, the comparative analysis is carried out to ensure that the result data in the study area is positive. By loading the actual drilling deviation data to the seismic work area, the seismic profile along the actual drilling trajectory is extracted from the 3D data volume, and the seismic reflection horizon corresponding to the synthetic record and the well trajectory seismic profile is calibrated, so that the wave group relationship and waveform characteristics are one-to-one corresponding. The multi well closure calibration is carried out by using single well and multi well profiles. On this basis, we can accurately track the reflection of target layer in multi direction and multi well connecting lines of $3 \mathrm{D}$ data to further understand the geological stratification ${ }^{[1]}$.

\subsection{Fault fine interpretation technology}

There are several methods for fault interpretation: first, for small faults that are easy to identify, the breakpoints are identified by using different display methods of waveform and density and seismic attribute volume; second, for the faults that are difficult to identify in conventional sections; the third is to identify the special attributes such as coherence cube and phase cosine, and to combine the faults by using coherence cube slice and dip angle detection;the fourth is the combination of earthquake and geology, and the application of geological stratification data to guide the fault

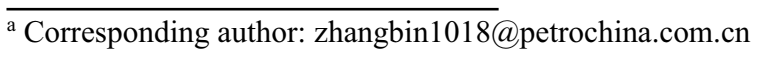


combination, so as to ensure the reasonable fault combination. At the same time, the synchronous browsing function of multi window and multi section display of interpretation system is applied. The position of horizon in the interpretation section is determined by the tie line section. The simultaneous display of adjacent multi sections is used to guide the interpretation of horizon and fault. The synchronous movement and display function of workstation is used to make the horizontal tracking and change of horizon and fault more reasonable and improve the interpretation speed and efficiency.

Coherent volume characterization is a common geophysical method used to identify faults. The special algorithm of coherence cube technology is to compare the similarity of local seismic waveforms through 3D data cube.The points with low coherence value are closely related to geological discontinuities such as faults, strata and special lithologic body boundaries.It is effective to identify faults by slicing the coherence data horizontally or along the layer.This time, we use the coherence cube to analyze the changes of underground geological structure, which is mainly used for fine interpretation of faults. In the interpretation, we can quickly browse the coherence data slices to establish the distribution mode of faults in the whole area. At the same time, in the process of plane combination of breakpoints, we can use the coherence data slice technology to guide the fault combination.Coherence technique is a means of discontinuity detection. The similarity between traces is calculated, the characteristics of small faults are highlighted, and the coherence volume is cut.Compared with conventional slices, these slices can better represent the fault and sedimentary characteristics.Coherent information is sensitive to fault detection,not only are the features of subsidence controlling faults prominent, but also the information of micro faults is abundant.The fault is divided by the methods of time slice of coherence volume and dip angle detection. The combination of matching and plane can not only improve the efficiency, but also browse the coherent volume slice and attribute volume slice to observe the change of fault strike and avoid different faults.Faults are artificially combined.The work flow of coherence volume analysis is divided into the following three steps: 1. Carry out comprehensive calibration for each well in the study area, and determine the accurate position of each standard layer,nonstandard layer and breakpoint, so as to determine the effective time window of data volume for seismic coherence calculation. 2 . Based on the block with high drilling density and clear fault system, the corresponding time window is opened in the target interval, and the statistical characteristics of coherence coefficient are analyzed for the records in the time window. Through continuous modification and verification, the time window and coherence parameters with good correlation are found. 3. Using the optimized coherence parameters, the seismic coherence calculation is carried out on the seismic data volume within the selected time window to generate coherent data volume, identify faults, and understand the distribution and combination of faults. The coherence analysis technology reduces the subjective judgment intervention and the influence of experience factors of the interpreter, and the interpretation results are more objective and reasonable. The resolution of faults is greatly improved and the working efficiency is greatly improved. It is very effective to identify small faults ${ }^{[2]}$.

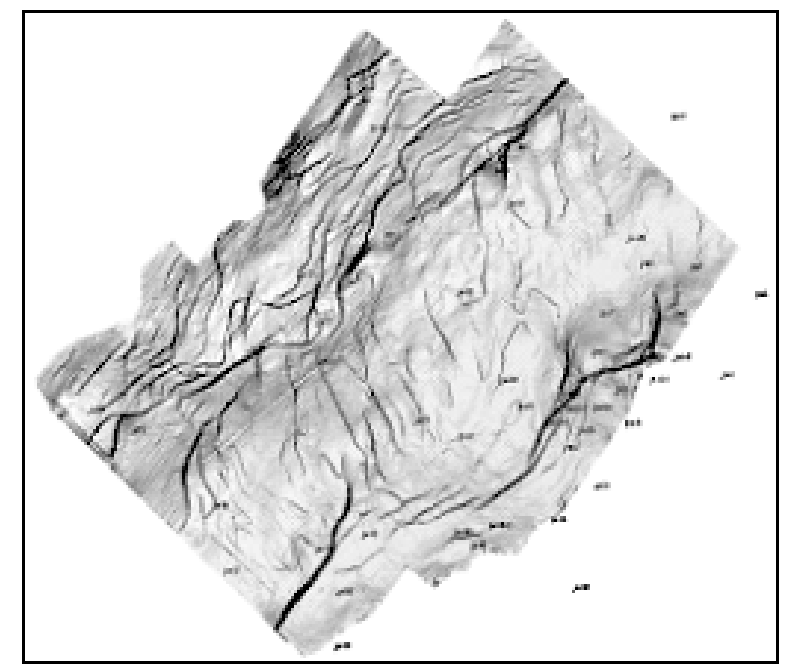

Figure 1. coherence plane of Nantun Formation in Northwest Beier Depression

Ant tracking technology can be used to identify small faults, and its algorithm is simply to optimize numerous paths, so as to find the best path. This algorithm can improve the accuracy of fault interpretation, help to interpret the faults that are not interpreted in the conventional section, and enhance the reliability of interpretation, Get the ant data body, and then extract various attributes for fault interpretation.

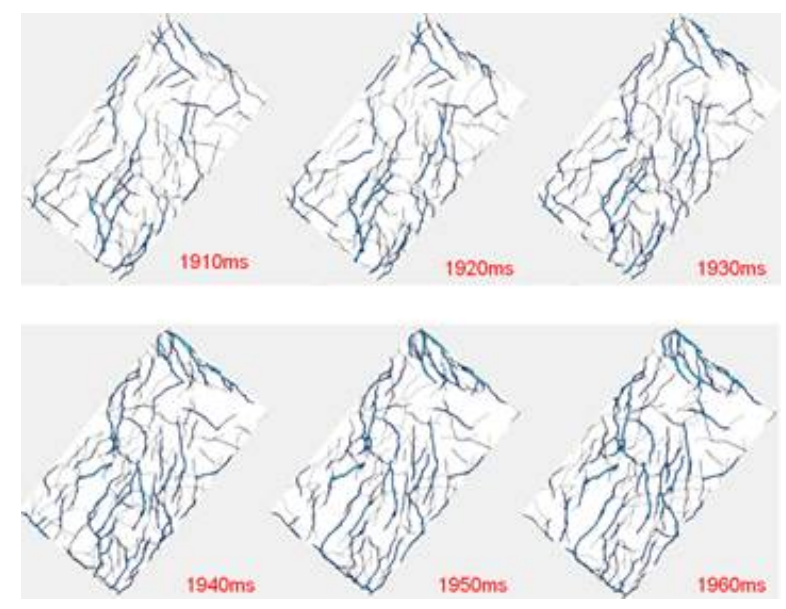

Figure 2. Ant body section of beizhongnantun formation

\subsection{Horizon stereoscopic interpretation}

Three dimensional interpretation is a comprehensive threed-imensional interpretation of the target interval in 3D space, starting from the well, along the vertical and horizontal sections, arbitrary sections, horizontal slices and even the whole data body. From the perspective of space, we can understand the structure and interpret the horizon. We can display the profile, special geological body, animation body, seat shaped section, interpretation 
horizon and section, which can improve the interpretation accuracy, This method can be used to accurately determine the structural shape and fault distribution of complex fault block area. The unified interpretation of horizons and faults is to track and compare horizons, and at the same time, to close the fault numbers one by one, so as to achieve accurate breakpoints of shallow, medium and deep horizons in the vertical direction, reasonable connection of faults, reasonable fault combination in the plane, and more accurately reflect its true shape, so as to avoid the intersection of hanging wall and footwall between upper and lower horizons. In the fine structural interpretation of complex fault block area in Beier Sag, more than 100 faults are interpreted, which ensures the consistency of fault interpretation and horizon interpretation and improves the accuracy of structural interpretation.At present, this technique is very practical for the description of complex fault block reservoir in the middle and later stage of exploration and development stage. It can straighten out the fracture relationship, combine the fault system reasonably, and in the high exploration and exploration area, combined with drilling data, it can also be used to determine the oil-water boundary of oil-water fault block. With the help of the high efficiency of the workstation, the interpretation accuracy of the block is greatly improved.

\subsection{Variable speed mapping technology}

The variable velocity mapping technology of "strata control + virtual well" is adopted for the first time in this mapping work, which improves the structural prediction accuracy of the target area.In the area with insufficient well control, according to the principle of "local equal time and depth", virtual well control is introduced to make the trend of depth structure map consistent with t0 map, and effectively improve the accuracy of structure mapping ${ }^{[3]}$.

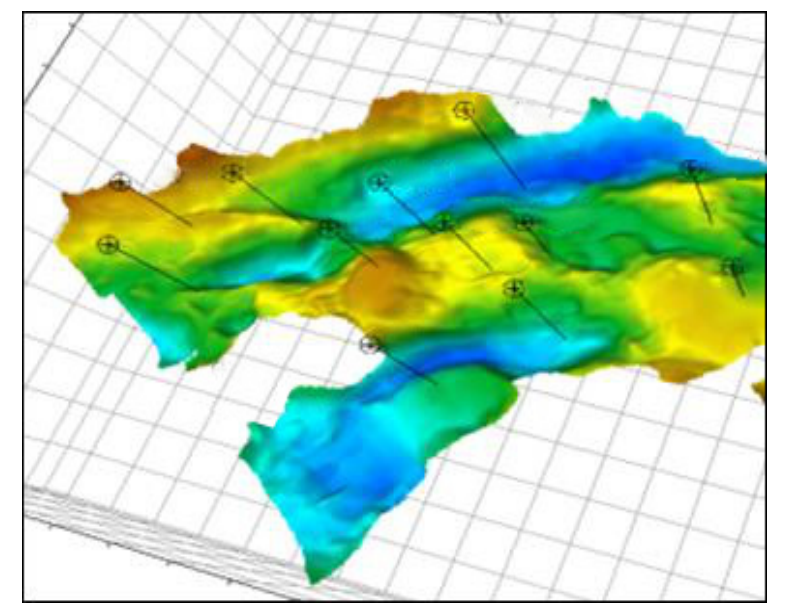

Figure 3. high precision 3D velocity field

\section{Fan body identification and reservoir}

\subsection{Optimization of inversion method for Nantun Formation in Beier Sag}

At present, there are two kinds of seismic inversion methods based on conventional 3D seismic data at home and abroad: one is model-based simulated annealing seismic inversion technology, which is independent of well data.Through the definition of inversion operator in frequency domain, the well data is expected to be output in the estimation of inversion operator. The inversion process is only deconvolution, so the inversion results maintain a good horizontal variation of sand body. The simulated annealing inversion results are based on the seismic data, and the inversion results are close to the seismic relative wave impedance attribute, which can be used for reference.As the inversion attribute controlled by seismic facies, it meets the requirements of fan characterization.Because of the multiple solutions of seismic inversion, it is difficult to select the most suitable tectonic geological model, which limits the advantages of this application ${ }^{[4]}$; secondly, geostatistics inversion technology is based on the principle of statistics. The prediction of thin interbeds is improved by interpolation with variogram function to obtain highfrequency components. However, the inversion results of this interpolation method are often quite different from the actual geological situation, and the geological concept is not strong, which makes the technology difficult. The promotion of the system is limited ${ }^{[5]}$.

The quality of seismic data in Beier Sag is low. At present, the dominant frequency is about $31 \mathrm{hz}$, and the low frequency components are generally distributed between $0 \sim 9 \mathrm{hz}$. The data of interpolation wells are obtained through the model;then, the intermediate frequency components between $9 \mathrm{~Hz}$ and $35 \mathrm{~Hz}$ are obtained by using seismic data;high frequency components of more than $45 \mathrm{~Hz}$ are obtained by cross well simulation, and phase control technology is highlighted. High frequency extraction is realized by statistical inversion.In this way, simulated annealing inversion and seismic waveform characteristic indication inversion are the core technologies. By segmented fusion of dominant frequency, the resolution of thin interbeds is improved, and the seismic bandwidth is improved, so as to achieve the purpose of depicting the fan body of fault basin. Through the practical application of this technology in Beier Sag, the vertical characterization of multi-stage fan body is realized, and the $10 \mathrm{~m}$ reservoir inside the fan body is predicted, and the reliability of the prediction result is verified by drilling. The frequency component ratio of simulated annealing inversion and Nantun Formation target layer seismic data is consistent in the frequency bandwidth $(9 \sim 35 \mathrm{~Hz})$, which is closer to the actual data than the waveform indication inversion. The simulated annealing inversion of 35 $80 \mathrm{~Hz}$ frequency component in SMI inversion results based on phase control is not available. The lowfrequency components of $0 \sim 9 \mathrm{hz}$ can be obtained by building a low-frequency model.By fusing the dominant frequencies of different frequency bands, the characterization of thin interbedded fan body under phase control can be improved.Figure 4 is the final inversion result, which can clearly show the distribution range of sandstone in the target layer.Above the target layer is the mudstone section, which forms the reflection 
feature of "mud in sand", so it presents the high resistance feature with a certain thickness under the background of low resistance, and the vertical resolution of sandstone has been significantly improved. The target layer is the lithologic combination of large section mudstone intercalated with siltstone and argillaceous siltstone, which shows the characteristics of high impedance, and the thin layer is in good agreement with the well characteristics. The inversion results are proved to be reliable.

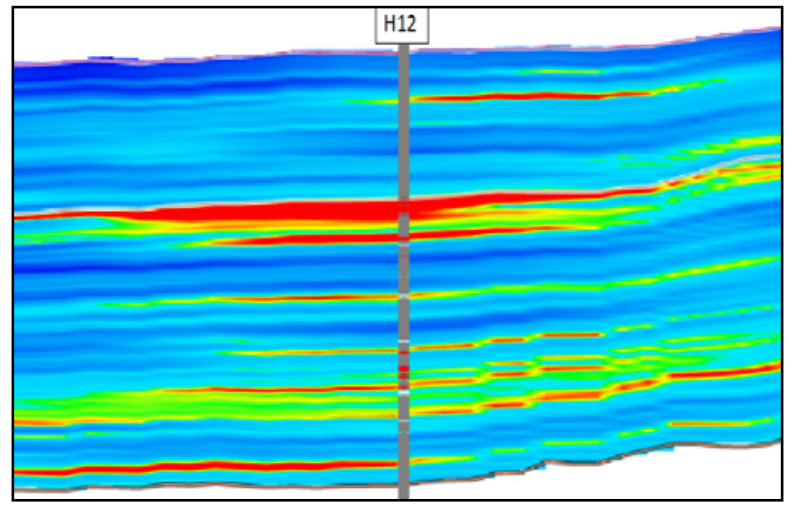

Figure 4. Inversion profile of fused frequency wave impedance through well $\mathrm{H} 12$

\subsection{Effect analysis}

Figure 5 shows the root mean square amplitude slice of inversion attributes along the target layer extracted by fusion frequency inversion, reflecting the spatial distribution characteristics and development range of H12 fan body. The fan body is developed from northwest to Southeast, showing a banded distribution. The fan body is developed in well $\mathrm{H} 12$ and H121, thin in well B71, and not in well H13; well H1, H20 and b40-1 are undeveloped, which proves the reliability of the prediction results and the operability of the prediction method.Through this method, 77 fans with an area of $480 \mathrm{~km} 2$ are predicted and depicted in Nantun Formation of Beier depression, and the reliability of prediction results is verified by drilling well $\mathrm{H} 12$.

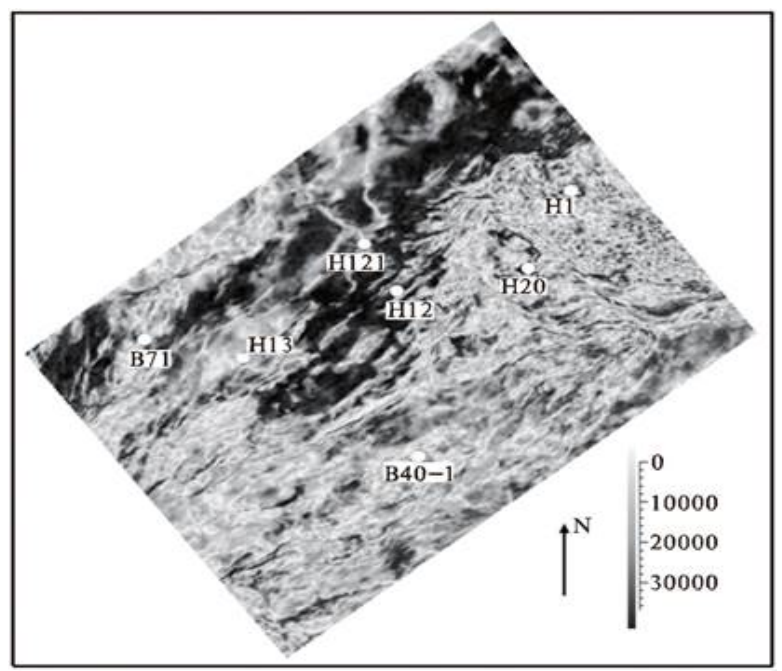

Figure 5. frequency fusion inversion Prediction Plan of well block H12 in Beier Sag

\section{4 conclusion}

In this paper, the time slicing technique, coherent volume description technique and ant tracking technique are used to explain the faults with different orders. The time slicing technique and coherent volume description technique are used to explain the faults with higher order combination, and the ant tracking technique is used to explain the faults with lower order combination. The accuracy of interpretation is improved.

The frequency fusion technology of different inversion methods plays an important role in fan body identification and internal reservoir characterization in complex fault basin. The full band seismic impedance inversion data volume formed by frequency fusion of simulated annealing inversion and seismic waveform indication inversion under facies control can realize the distribution prediction of favorable reservoir $(10 \mathrm{~m})$ in fan body.It provides ideas for exploration and target optimization of complex fault basin.

\section{References}

1. Ma Lijuan, Jin Zhijun. (2005) Fine interpretation of complex fault block. J. Petroleum geophysical exploration, 40(6): 688-692.

2. She Deping, Cao Hui, Guo Quanshi. (2000) Fine interpretation using $3 \mathrm{D}$ coherence technique. J. Petroleum geophysical prospecting, 39 (2): 83-88.

3. Zhang Yonghua, Ren junzhan, Yan Yisheng. (2010) Micro structural interpretation and variable velocity mapping method. J. Petroleum geophysical exploration, 49 (2): 176-181.

4. Wang Xiangwen, Liu Hong, Teng Binbin. (2012) Application of geostatistical inversion technique in thin reservoir pre-processing Application in geological survey. J. Petroleum and natural gas geology, 33(5): 730-735.

5. Sun Si min, Peng Shi MI. (2007) Geostatistical inversion method and its application in thin sand body predictionJournal of Xi'an University of petroleum. J. Natural Science Edition, 22(1): 141148. 